\title{
Características Morfológicas de Gramas em Resposta à APLiCAÇÃo de TrineXAPAC-ETHYL ${ }^{1}$
}

\author{
Morphological Characteristics of Turf Grasses in Response to Trinexapac-Ethyl Application
}

\begin{abstract}
COSTA, N.V. ${ }^{2}$, MARTINS, D. ${ }^{3}$, RODELLA, R.A. ${ }^{4}$, RODRIGUES, A.C.P. ${ }^{5}$ e CARDOSO, L.A. ${ }^{5}$
RESUMO - O presente trabalho teve como objetivo avaliar os efeitos da aplicação de duas doses de trinexapac-ethyl sobre a morfologia das espécies de gramas São Carlos (Axonopus compressus), Batatais (Paspalum notatum), Santo Agostinho (Stenotaphrum secundatum) e Esmeralda (Zoysia japonica). Os gramados foram cortados à altura de $3 \mathrm{~cm}$ no início do experimento e 20 dias depois. Após cada corte, foram realizadas duas aplicações sequenciais de trinexapac-ethyl nas doses de 56,5 + 56,5 e 113,0 $+113,0 \mathrm{~g} \mathrm{ha}^{-1}$, além de uma testemunha sem aplicação, para cada espécie avaliada. O delineamento experimental utilizado foi o de blocos ao acaso com quatro repetições. A redução do crescimento foi avaliada por meio da altura das plantas. Semanalmente, o número e altura de inflorescências foram avaliados por amostragem, realizada em $0,25 \mathrm{~m}^{2}$ no centro das parcelas; no final do experimento, avaliou-se a massa seca total. A aplicação do trinexapac-ethyl retardou o crescimento vegetativo e a emissão das inflorescências, assim como não provocou danos aparentes nos gramados. O uso do trinexapac-ethyl nos gramados avaliados pode reduzir a necessidade de cortes em até 55 dias após a segunda aplicação.
\end{abstract}

Palavras-chave: regulador vegetal, Axonopus compressus, Paspalum notatum, Stenotaphrum secundatum e Zoysia japonica.

\begin{abstract}
The aim of this research was to evaluate the effects of the sequential application of two rates of trinexapac-ethyl on the morphology of the following turf grass species: Broadleaf Carpetgrass (Axonopus compressus), Bahiagrass (Paspalum notatum), St. Augustinegrass (Stenotaphrum secundatum) and Korean Lawngrass (Zoysia japonica). The treatments used were trinexapac-ethyl with two sprays applied at 20 day interval at two different rates $\left(56.5+56.5\right.$ and $\left.113.0+113.0 \mathrm{~g} \mathrm{ha}^{-1}\right)$ and a control without spraying, for each species. The turf grasses were cut at the height of $3 \mathrm{~cm}$, and sprayed after the treatments. Twenty days after the first treatment application, the plots were cut again and the second treatment application was carried out. The experiment was arranged in a completely randomized block design with four replications. Growth reduction was evaluated based on the height of the plants. Inflorescence number and height were weekly evaluated by sampling, carried out in $0.25 \mathrm{~m}^{2}$ in the center of the plots. Total dry matter was evaluated at the end of the experiment. Ttrinexapac-ethyl application delayed growth and inflorescence emission and did not cause any visual damage to the turf grasses. Trinexapac-ethyl application can reduce the need of cuts for a period up to 55 days after the second application.
\end{abstract}

Keywords: growth regulator, Axonopus compressus, Paspalum notatum, Stenotaphrum secundatum and Zoysia japonica.

Recebido para publicação em 26.1.2008 e na forma revisada em 18.2.2009.

2 Doutor em Agronomia/Agricultura, <neumarciovc@hotmail.com>; ${ }^{3}$ Professor Adjunto do Dep. de Produção Vegetal da FCA-UNESP, Fazenda Lageado, Caixa Postal 237, 18603-970 Botucatu-SP; ${ }^{4}$ Professor Adjunto do Dep. de Botânica, Instituto de Biociências de Botucatu-UNESP, Botucatu-SP, ${ }^{5}$ Doutorando em Agronomia do Dep. de Produção Vegetal da FCA-UNESP, Botucatu-SP 


\section{INTRODUÇÃO}

A palavra GRAMA tem sua origem na denominação da fam[ilia botânica das gramíneas (Gramineae $=$ Poaceae $)$, que engloba mais de 10.000 espécies dispersas pelo mundo, sendo que as mais importantes servem como alimentação humana, como o arroz, milho e o trigo, ou como alimentação animal como as brachiarias, havendo outras que são usadas até em construções, como os bambus, entretanto, menos de 50 espécies das gramíneas podem ser utilizadas na formação de gramados (Watson \& Dallwitz, 1992).

Entre as espécies de gramas adaptadas às condições climáticas brasileiras, destacam-se: São Carlos (Axonopus compressus), Batatais ou Bahia (Paspalum notatum), Santo Agostinho (Stenotaphrum secundatum) e Esmeralda (Zoysia japonica) (Pimenta, 2003).

Essas espécies podem ser utilizadas em obras públicas, parques industriais, áreas esportivas, assim como em áreas residenciais. Contudo, em todos esses usos o corte destacase como o principal fator do custo de manutenção para manter a altura adequada dos gramados (Maciel et al., 2006).

Os reguladores vegetais utilizados em gramas reduzem a estatura das plantas sem prejudicar a densidade ou causar dano visivel ao gramado, como pontos necróticos de fitotoxicidade, descoloração ou afinamento, mantendo a alta qualidade da área tratada. Dessa forma, podem diminuir o número de operações de corte que são realizadas durante o período de crescimento, compreendido entre a primavera e o verão, atuando indiretamente como potencial componente na redução de custos com mão-de-obra, combustivel e equipamentos (Johnson, 1992; Jiang \& Fry, 1998; Lickfeldt et al., 2001; Freitas et al., 2002).

Assim, a aplicação de reguladores vegetais, além de possibilitar a redução da freqüência de corte e os custos de manutenção dos gramados, pode funcionar como uma ferramenta alternativa e menos agressiva para o manejo da altura dos gramados de diferentes propósitos (Ferrell et al., 2003). Entre os reguladores vegetais com grande potencial em utilização para gramados, destaca-se o trinexapac-ethyl.

A aplicação de reguladores vegetais no Brasil ainda é considerada restrita, sendo eles utilizados apenas em algumas culturas, como algodão, cana-de-açúcar, certas frutíferas e na produção de flores. Mais insignificante ainda é o seu uso para o manejo de gramados, seja em áreas esportivas, residenciais, industriais, lazer ou margens de rodovias (Velini, 2003). Dessa maneira, vários compostos sintéticos podem ser utilizados para modificar o hábito de crescimento das plantas, quando aplicados exogenamente (Grossmann et al., 1987).

Diversos trabalhos em que se avalia o regulador vegetal trinexapac-ethyl constataram a inibição do crescimento, com eficiência de quatro a seis semanas após o tratamento, em várias espécies de grama (Johnson, 1992, 1993, 1994; Fagerness \& Penner, 1998a,b; Ervin \& Koski, 2001; Freitas et al., 2002). Pertencente ao grupo químico das cicloexanodionas, o trinexapac-ethyl apresenta estrutura similar à dos herbicidas graminicidas sethoxydim e clethodim; contudo, seu mecanismo de ação está relacionado com a inibição da biossintese de giberelinas e ocorre na terceira fase da sintese das giberelinas, no citoplasma celular, reduzindo o nivel de giberelinas ativas, principalmente $\mathrm{GA}_{1}$, por meio da redução da atividade da enzima GA $_{20}$ $3 \beta$-hidroxilase. Assim, a inibição da atividade desta enzima provavelmente resulta da competição entre o regulador vegetal e o 2oxoglutarato pelo co-substrato $\mathrm{Fe}^{+2}$ / ascorbatodependente dioxigenase (Adams et al., 1992; Rodrigues et al., 2004). A inibição do crescimento da planta é decorrente da redução do alongamento celular e não da divisão celular (Ervin \& Koski, 2001).

Em condições brasileiras, destacam-se os trabalhos realizados por Freitas et al. (2002) em grama Paspalum notatum e por Maciel et al. (2006) em grama Cynodon dactylon. Os resultados obtidos por esses pesquisadores evidenciaram relação direta entre o aumento das doses de trinexapac-ethyl e a redução dos parâmetros de crescimento vegetativo e reprodutivo, sem ocorrer descoloração das folhas dos gramados. Entretanto, estudos morfológicos de espécies de grama, principalmente relacionados com o efeito da aplicação de reguladores vegetais de crescimento, são escassos no Brasil.

O presente trabalho teve por objetivo avaliar os efeitos da aplicação sequencial de duas 
doses de trinexapac-ethyl sobre a morfologia da planta das espécies de grama $A$. compressus, $P$. notatum, $S$. secundatum e $Z$. japonica.

\section{MATERIAL E MÉTODOS}

O estudo foi conduzido no Núcleo de Pesquisas Avançadas em Matologia - NUPAM, do Departamento de Produção Vegetal da Faculdade de Ciências Agronômicas de Botucatu/ UNESP. O local do experimento apresenta as seguintes coordenadas geográficas: latitude de $22^{\circ} 07^{\prime} 56^{\prime \prime} \mathrm{S}$, longitude de $74^{\circ} 66^{\prime} 84^{\prime}$ 'W Gr. e altitude de $762 \mathrm{~m}$. Foram avaliadas as espécies de grama São Carlos (Axonopus compressus), Batatais (Paspalum notatum), Santo Agostinho (Stenotaphrum secundatum) e Esmeralda (Zoysia japonica). As quatro espécies de grama foram plantadas em tabuleiros individuais e estavam com 26 meses de idade no momento da aplicação dos tratamentos. Em cada espécie de grama foi utilizado o delineamento experimental de blocos ao acaso com quatro repetições. As parcelas foram constituídas de $2 \mathrm{~m}$ de largura por $2 \mathrm{~m}$ de comprimento.

Os tratamentos foram aplicados em 3/2/ 2006, sendo realizadas duas aplicações sequenciais de trinexapac-ethyl (Primo Maxx ${ }^{\mathrm{TM}}$, $113,0 \mathrm{~g} \mathrm{~L}^{-1}$ - produto comercial) nas doses de $56,5+56,5$ e $113,0+113,0 \mathrm{~g} \mathrm{ha}^{-1}$, além de uma testemunha sem aplicação para cada espécie avaliada, com intervalo de 20 dias entre as aplicações.

Os gramados foram cortados com um aparador de grama motorizado à altura de $3 \mathrm{~cm}$ e, em seguida, foram realizadas as aplicações dos tratamentos. As aplicações ocorreram no período da manhã, em condições de temperatura de $24{ }^{\circ} \mathrm{C}$, solo úmido e umidade relativa do ar em torno de $80 \%$. Foi utilizado um pulverizador costal, pressurizado a $\mathrm{CO}_{2}$ e munido de barra com quatro pontas de jato plano XR 11002 VS, espaçados entre si de $50 \mathrm{~cm}$, sendo o consumo de calda equivalente a $200 \mathrm{~L} \mathrm{ha}^{-1}$.

Durante a aplicação, as parcelas foram protegidas lateralmente com biombos de lona plástica, com o objetivo de evitar deriva de calda pulverizada para as parcelas adjacentes. Aos 20 dias após a primeira aplicação dos tratamentos, as parcelas foram novamente aparadas à altura de $3 \mathrm{~cm}$ e realizou-se a segunda aplicação dos tratamentos.
Os dados climáticos (temperatura minima, temperatura máxima, temperatura média, insolação, precipitação pluvial e unidade relativa do ar) encontram-se nas Figuras 1 e 2. Os caracteres morfológicos avaliados foram: altura de planta, número e comprimento de inflorescência e massa seca. As avaliações relativas ao número e comprimento das inflorescências foram feitas semanalmente, até os 28 dias após a segunda aplicação. O número de inflorescências foi avaliado por amostragem, com auxílio de um quadro de $0,25 \mathrm{~m}^{2}$ colocado no centro das parcelas, e o comprimento das inflorescências foi determinado com auxílio de uma régua graduada, medindo-se a distância entre o solo e as extremidades delas, amostrandose apenas a inflorescência mais alta do centro de cada parcela. As avaliações da altura de planta foram feitas aos $7,14,21,28,42,56$ e 70 dias após a segunda aplicação, com auxílio de duas réguas graduadas, sendo posicionadas, no momento da avaliação, perpendicular e horizontalmente ao solo (em forma de cruz)

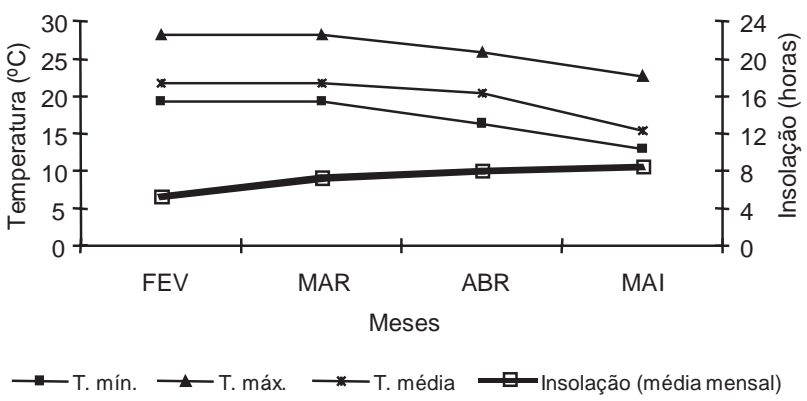

Figura 1 - Médias mensais das temperaturas mínima (T. mín.), máxima (T. máx.) e média (T. média), em ${ }^{\circ} \mathrm{C}$, e da insolação (em horas), em Botucatu-SP, durante o período de condução do experimento. Botucatu-SP, 2006.

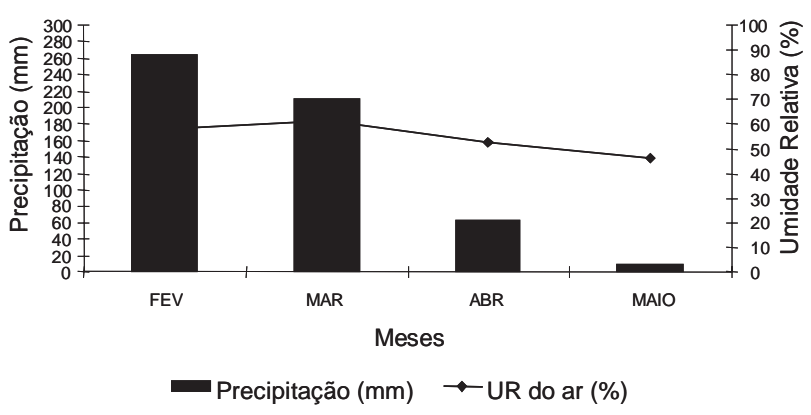

Figura 2 - Precipitações pluviais e as médias mensais da umidade relativa do ar, em Botucatu-SP, durante o período de condução do experimento. Botucatu-SP, 2006.

Planta Daninha, Viçosa-MG, v. 27, n. 1, p. 113-122, 2009 
em apenas um ponto de cada parcela, medindo-se a distância entre o solo e a extremidade da folha mais alta. A régua na horizontal tinha como função facilitar a determinação da folha mais alta do centro da parcela.

A massa seca da parte aérea produzida pelas gramas foi determinada no final do experimento, coletando-se a massa vegetal produzida na parcela após o corte da grama a $3 \mathrm{~cm}$ de altura, com auxílio de um aparador de grama motorizado, para posterior secagem em estufa de circulação forçada de ar a $65^{\circ} \mathrm{C}$, até massa constante.

Os dados de porcentagem de redução do número e do comprimento de inflorescência, da altura e do acúmulo de massa seca de plantas foram obtidos considerando as testemunhas de cada espécie avaliada como referência, respectivamente.

Os dados de altura de planta $(\mathrm{cm})$, número de inflorescências $\left(\mathrm{n}^{\circ} \mathrm{m}^{-2}\right)$ e comprimento de inflorescências $(\mathrm{cm})$ foram analisados por regressão polinomial, bem como pelo modelo sigmoidal:

$$
y=a+\frac{b}{\left[1+(x / c)^{d}\right]}
$$

em que:

$y$ = variável resposta (altura de planta, número de inflorescências e comprimento de inflorescências);

$x=$ dias após a segunda aplicação;

$a=$ valor mínimo no início da avaliação da variável analisada;

$b=$ diferença entre o valor máximo e o mínimo da variável analisada;

$c=\mathrm{n}$ o de dias em que houve $50 \%$ de redução do valor máximo da variável analisada; e

$d=$ declividade da curva.

Os caracteres quantitativos da morfologia das plantas de grama também foram submetidos à análise de variância; os dados originais, em número, foram transformados utilizandose $\sqrt{x+0,5}$, respectivamente, sendo analisados estatisticamente, empregando-se o teste de média LSD a $5 \%$ de probabilidade.

\section{RESULTADOS E DISCUSSÃO}

O período de condução do experimento (fevereiro a abril) coincidiu com o verão e parte do outono, sendo caracterizado por temperaturas médias variando de 15 a $24^{\circ} \mathrm{C}$, além da ocorrência de precipitações regulares (Figuras 1 e 2). Essas condições proporcionaram condição favorável ao crescimento das espécies avaliadas; a partir do mês de maio, ou seja, no final do outono, com a redução da temperatura, da luminosidade e da umidade no solo, como consequência da redução da ocorrência de chuvas, a taxa de crescimento das plantas reduziu drasticamente, caracterizando o início do periodo desfavorável ao crescimento dos gramados formados por espécies tropicais, com mecanismo fotossintético $\mathrm{C}_{4}$.

Podem-se observar, na Figura 3, as equações de regressão referentes ao número de inflorescência apresentado pelas quatro espécies de grama avaliadas, em função da aplicação de trinexapac-ethyl, após a segunda aplicação dos tratamentos.

Dessa forma, verificou-se que as testemunhas de $A$. compressus, $S$. secundatum e Z. japonica iniciaram a emissão das inflorescências a partir dos 14 dias após a segunda aplicação do regulador vegetal, enquanto para a espécie $P$. notatum verificou-se emissão de inflorescências já na avaliação realizada aos sete dias após o corte, para a segunda aplicação do regulador vegetal. No entanto, ambas as doses de trinexapac-ethyl retardaram a emissão das inflorescências por um período de 14, 21 e 21 dias após a segunda aplicação do regulador vegetal em A. compressus, S. secundatum e $Z$. japonica, respectivamente (Figura 3).

Ressalta-se que os tratamentos que emitiram inflorescência precocemente, a partir dos sete dias após a segunda aplicação de trinexapac-ethyl, tenderam a apresentar comportamento semelhante ao do modelo de regressão polinomial, a exemplo dos dados da testemunha de $S$. secundatum e da dose de $56,5 \mathrm{~g}^{-1}$ de $A$. compressus e $P$. notatum, bem como da dose de 113,0 $\mathrm{g} \mathrm{ha}^{-1}$ de $P$. notatum e $Z$. japonica. Contudo, os tratamentos que emitiram inflorescências tardiamente, a partir dos 14 dias após a segunda aplicação, tenderam a apresentar comportamento semelhante ao de modelo de regressão sigmoidal, a exemplo 
dos dados das doses de 56,5 e 113,0 $\mathrm{g} \mathrm{ha}^{-1}$ de $A$. compressus e da dose de $56,5 \mathrm{~g} \mathrm{ha}^{-1}$ de S. secundatum,
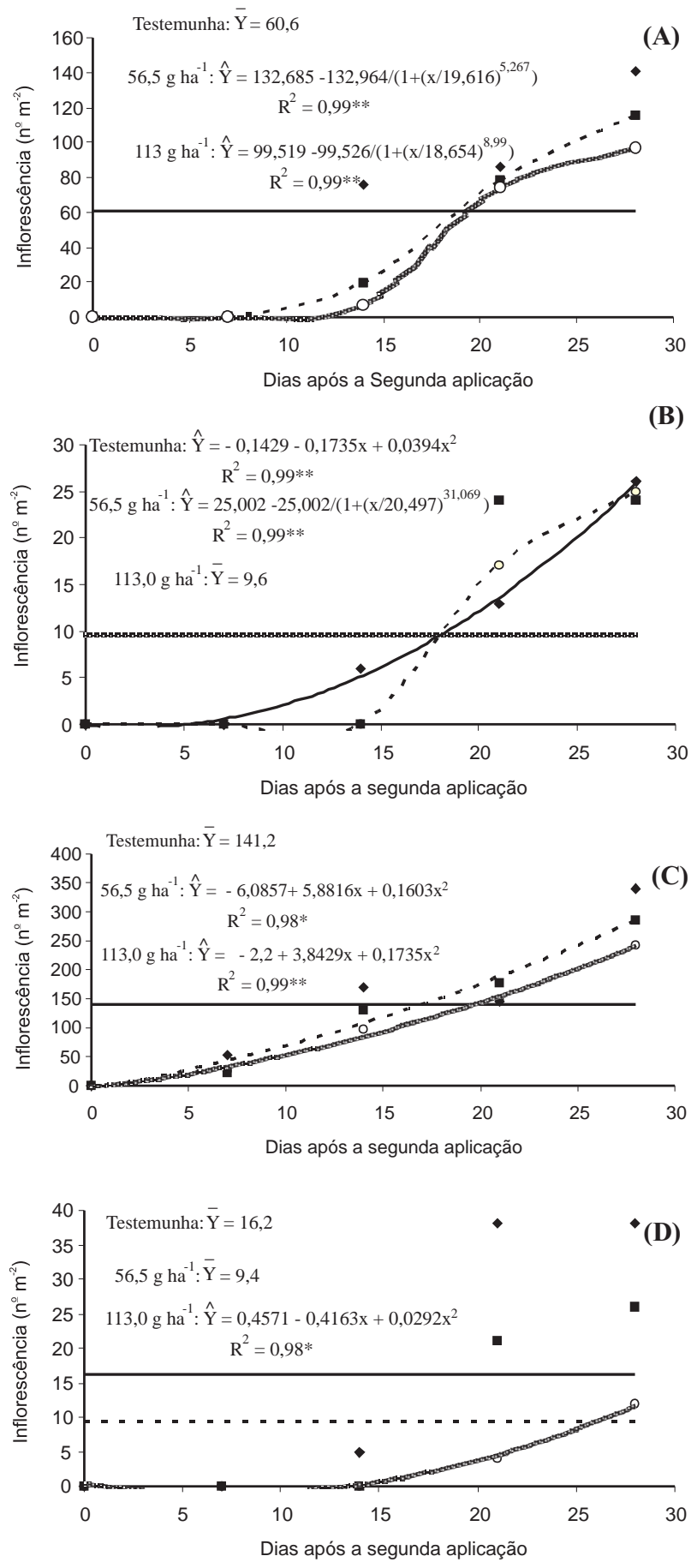

Figura 3 - Número de inflorescências em diferentes épocas de avaliação, após a segunda aplicação de trinexapac-ethyl, para as espécies Axonopus compressus (A), Stenotaphrum secundatum (B), Paspalum notatum (C) e Zoysia japonica (D). Botucatu-SP - 2006
Para $P$. notatum, a partir de sete dias após a segunda aplicação de trinexapac-ethyl, já havia necessidade da realização de uma roçada para eliminação das inflorescências e melhoria do aspecto visual do gramado (Figura 3C). Freitas et al. (2002) citam a alta capacidade de emissão de inflorescência de $P$. notatum como um dos principais problemas no manejo de gramados formados com essa espécie. Ressalta-se que a partir dos 28 dias após a segunda aplicação todas as inflorescências das quatro gramas avaliadas começaram o processo de senescência.

Vários trabalhos ressaltam que a aplicação de trinexapac-ethyl pode retardar e/ou reduzir a emissão das inflorescências e prolongar o aspecto visual do gramado, sem que haja a necessidade de uma nova roçada (Nelson et al., 1977; Bush et al., 1998; Fagerness \& Penner, 1998a, b; Freitas et al., 2002).

Segundo Bush et al. (1998), a dose de $0,48 \mathrm{~kg} \mathrm{ha}^{-1}$ de trinexapac-ethyl reduziu a emissão de inflorescência em Axonopus affins, pelo período de seis semanas, em $29 \%$. A aplicação de trinexapac-ethyl na dose de $0,287 \mathrm{~kg} \mathrm{ha}^{-1}$ para a grama Poa pratensis e de $0,382 \mathrm{~kg} \mathrm{ha}^{-1}$ para Lolium perene proporcionou reduções na emissão de inflorescência em ambas as espécies de 43 e 57\%, respectivamente, tendo seus efeitos dissipados após quatro semanas da aplicação (Fagerness \& Penner, 1998a).

O trinexapac-ethyl demonstrou tendência para promover reduções do número de inflorescências acima de $15 \%$ nos gramados, exceto para a grama $S$. secundatum, que apresentou redução inferior a $8 \%$, independentemente da dose utilizada (Tabela 1).

Na Figura 4 são apresentadas as equações de regressão ajustadas para os dados de comprimento de inflorescências em diferentes épocas de avaliação, após a segunda aplicação de trinexapac-ethyl nas espécies de gramas avaliadas. Pode-se observar que, semelhante aos dados da Figura 3, as doses utilizadas do regulador vegetal que proporcionaram emissão das inflorescências a partir dos 14 dias após a segunda aplicação também apresentaram desenvolvimento menor das inflorescências que o das testemunhas. Outro ponto importante que pode ter contribuído com o baixo 
crescimento das inflorescências é que a emissão foi próximo ao período de senescência (28 dias após a segunda aplicação).

Com relação às medias do comprimento de inflorescências aos 28 dias após a segunda aplicação, não houve efeito do trinexapac-ethyl apenas para $Z$. japonica (Tabela 1); entretanto, trata-se de uma espécie que produz reduzido número de inflorescências, se comparada a A. compressus e $P$. notatum, e possui comprimento de inflorescência bem inferior ao de $S$. secundatum e $P$. notatum, tornando o efeito visual das inflorescências discreto. As espécies $S$. secundatum, $P$. notatum e $Z$. japonica apresentaram, na menor dose, inflorescências $68,9,47,6$ e 40,0\% mais curtas em relação à testemunha não aplicada, respectivamente. Já para A. compressus observaram-se inflorescências até 20,24 e 24,09\% mais curtas que as da testemunha, para as doses de 56,5 e $113,0 \mathrm{~g} \mathrm{ha}^{-1}$, respectivamente (Tabela 1 ).

O trinexapac-ethyl é um regulador vegetal que está sendo avaliado há alguns anos para o manejo de gramados, porém possui baixa eficiência na supressão das inflorescências (Johnson, 1994). Esse fato pode ser explicado devido ao trinexapac-ethyl pertencer aos reguladores do tipo II, que interferem na

Tabela 1 - Valores médios das características morfológicas das plantas das quatro espécies de gramas, após a aplicação de trinexapac-ethyl. Botucatu-SP - 2006

\begin{tabular}{|c|c|c|c|c|c|c|c|}
\hline \multirow{2}{*}{$\mathrm{N}^{\mathrm{o}}$} & \multirow{2}{*}{ Caracteres } & \multicolumn{6}{|c|}{ Axonopus compressus } \\
\hline & & Testemunha & $56,5 \mathrm{~g} \mathrm{ha}^{-1}$ & $113,0 \mathrm{~g} \mathrm{ha}^{-1}$ & $\mathrm{~F}_{\text {(Tratamentos) }}$ & D.M.S. & $\mathrm{CV}(\%)$ \\
\hline 1. & Número de inflorescência $\left(\mathrm{n} \mathrm{m}^{-2}\right)^{1 / 2 !}$ & 141,00 & 115,00 & 97,00 & $0,955^{\mathrm{ns}}$ & 2,164 & 23,50 \\
\hline 2. & Comprimento da inflorescência $(\mathrm{cm})^{\frac{1}{}}$ & $9,88 \mathrm{a}$ & $7,88 \mathrm{~b}$ & $7,50 \mathrm{~b}$ & $6,306^{*}$ & 2,019 & 13,59 \\
\hline 3. & Altura da planta $(\mathrm{cm})^{3 /}$ & $6,50 \mathrm{a}$ & $4,00 \mathrm{~b}$ & $3,88 \mathrm{~b}$ & $54,600 * *$ & 0,645 & 7,58 \\
\hline 4. & Massa seca $\left(\mathrm{kg} \mathrm{ha}^{-1}\right)^{3 /}$ & $611,25 \mathrm{a}$ & $386,25 \mathrm{ab}$ & $233,75 \mathrm{~b}$ & $5,300 *$ & 285,45 & 40,20 \\
\hline \multirow{2}{*}{$\mathrm{N}^{\mathrm{o}}$} & \multirow{2}{*}{ Caracteres } & \multicolumn{6}{|c|}{ Paspalum notatum } \\
\hline & & Testemunha & $56,5 \mathrm{~g} \mathrm{ha}^{-1}$ & $113,0 \mathrm{~g} \mathrm{ha}^{-1}$ & $\mathrm{~F}_{\text {(Tratamentos) }}$ & D.M.S. & $\mathrm{CV}(\%)$ \\
\hline 1. & Número de inflorescência $\left(\mathrm{n} \mathrm{m}^{-2}\right)^{1 /, 2 /}$ & 340,00 & 286,00 & 243,00 & $3,089^{\text {ns }}$ & 1,454 & 9,91 \\
\hline 2. & Comprimento da inflorescência $(\mathrm{cm})^{\frac{1}{}}$ & 23,38 a & $12,25 \mathrm{~b}$ & $7,25 \mathrm{c}$ & $140,493 * *$ & 2,430 & 9,80 \\
\hline 3. & Altura da planta $(\mathrm{cm})^{3 /}$ & $15,00 \mathrm{a}$ & $10,75 \mathrm{a}$ & $5,88 \mathrm{~b}$ & $13,090 * *$ & 4,306 & 23,52 \\
\hline 4. & Massa seca $\left(\mathrm{kg} \mathrm{ha}^{-1}\right)^{3 /}$ & $2.136,25 \mathrm{a}$ & $1.101,25 \mathrm{~b}$ & $668,75 \mathrm{~b}$ & $13,980 * *$ & 714,74 & 31,73 \\
\hline \multirow{2}{*}{$\mathrm{N}^{\mathrm{o}}$} & \multirow{2}{*}{ Caracteres } & \multicolumn{6}{|c|}{ Stenotaphrum secundatum } \\
\hline & & Testemunha & $56,5 \mathrm{~g} \mathrm{ha}^{-1}$ & $113,0 \mathrm{~g} \mathrm{ha}^{-1}$ & $\mathrm{~F}_{\text {(Tratamentos) }}$ & D.M.S. & $\mathrm{CV}(\%)$ \\
\hline 1. & Número de inflorescência $\left(\mathrm{n} \mathrm{m}^{-2}\right)^{1 / 2,21}$ & 26,00 & 25,00 & 24,00 & $0,277^{\mathrm{ns}}$ & 1,040 & 24,44 \\
\hline 2. & Comprimento da inflorescência $(\mathrm{cm})^{\frac{1}{}}$ & $11,25 \mathrm{a}$ & $3,50 \mathrm{~b}$ & $5,00 \mathrm{ab}$ & $5,016^{*}$ & 6,351 & 55,75 \\
\hline 3. & Altura da planta $(\mathrm{cm})^{3 /}$ & $5,25 \mathrm{a}$ & $3,75 \mathrm{~b}$ & $3,25 \mathrm{~b}$ & $23,400 * *$ & 0,645 & 8,77 \\
\hline 4. & Massa seca $\left(\mathrm{kg} \mathrm{ha}^{-1}\right)^{\frac{3}{}}$ & 453,75 & 263,75 & 253,75 & $4,389^{\mathrm{ns}}$ & 186,2 & 33,23 \\
\hline \multirow{2}{*}{$\mathrm{N}^{\mathrm{o}}$} & \multirow{2}{*}{ Caracteres } & \multicolumn{6}{|c|}{ Zoysia japonica } \\
\hline & & Testemunha & $56,5 \mathrm{~g} \mathrm{ha}^{-1}$ & $113,0 \mathrm{~g} \mathrm{ha}^{-1}$ & $\mathrm{~F}_{\text {(Tratamentos) }}$ & D.M.S. & $\mathrm{CV}(\%)$ \\
\hline 1. & Número de inflorescência $\left(\mathrm{n} \mathrm{m}^{-2}\right)^{1 /, 2 l}$ & 38,00 & 26,00 & 12,00 & $3,516^{\mathrm{ns}}$ & 1,251 & 29,12 \\
\hline 2. & Comprimento da inflorescência $(\mathrm{cm})^{\frac{1}{}}$ & 6,25 & 3,75 & 2,38 & $3,500^{\mathrm{ns}}$ & 3,738 & 49,85 \\
\hline 3. & Altura da planta $(\mathrm{cm})^{3 /}$ & $7,88 \mathrm{a}$ & $4,00 \mathrm{~b}$ & $3,50 \mathrm{c}$ & $100,500 * *$ & 0,816 & 8,84 \\
\hline 4. & Massa seca $\left(\mathrm{kg} \mathrm{ha}^{-1}\right)^{3 /}$ & $1.921,25 \mathrm{a}$ & $656,25 \mathrm{~b}$ & $646,25 \mathrm{~b}$ & $15,747 * *$ & 639,44 & 34,39 \\
\hline
\end{tabular}

${ }^{1 /}$ Dados originais transformados em $\sqrt{x+0,5} \cdot{ }^{2 /}$ significativo a $1 \%$ de probabilidade; ${ }^{3 /}$ significativo a $5 \%$ de probabilidade; $\mathrm{e}^{\text {ns }}$ não-significativo. Médias seguidas de mesma letra, nas linhas, não diferem estatisticamente entre si a $5 \%$ de probabilidade pelo teste LSD 
biossintese de giberelina e na redução da elongação celular e não na divisão celular, a exemplo dos reguladores do tipo I, como amidochlor, mefluidide e hidrazida maleica (Davis \& Curry, 1991 citado por Velini, 2003).

Com relação à altura de plantas, verificaram-se diferenças para as quatro espécies de
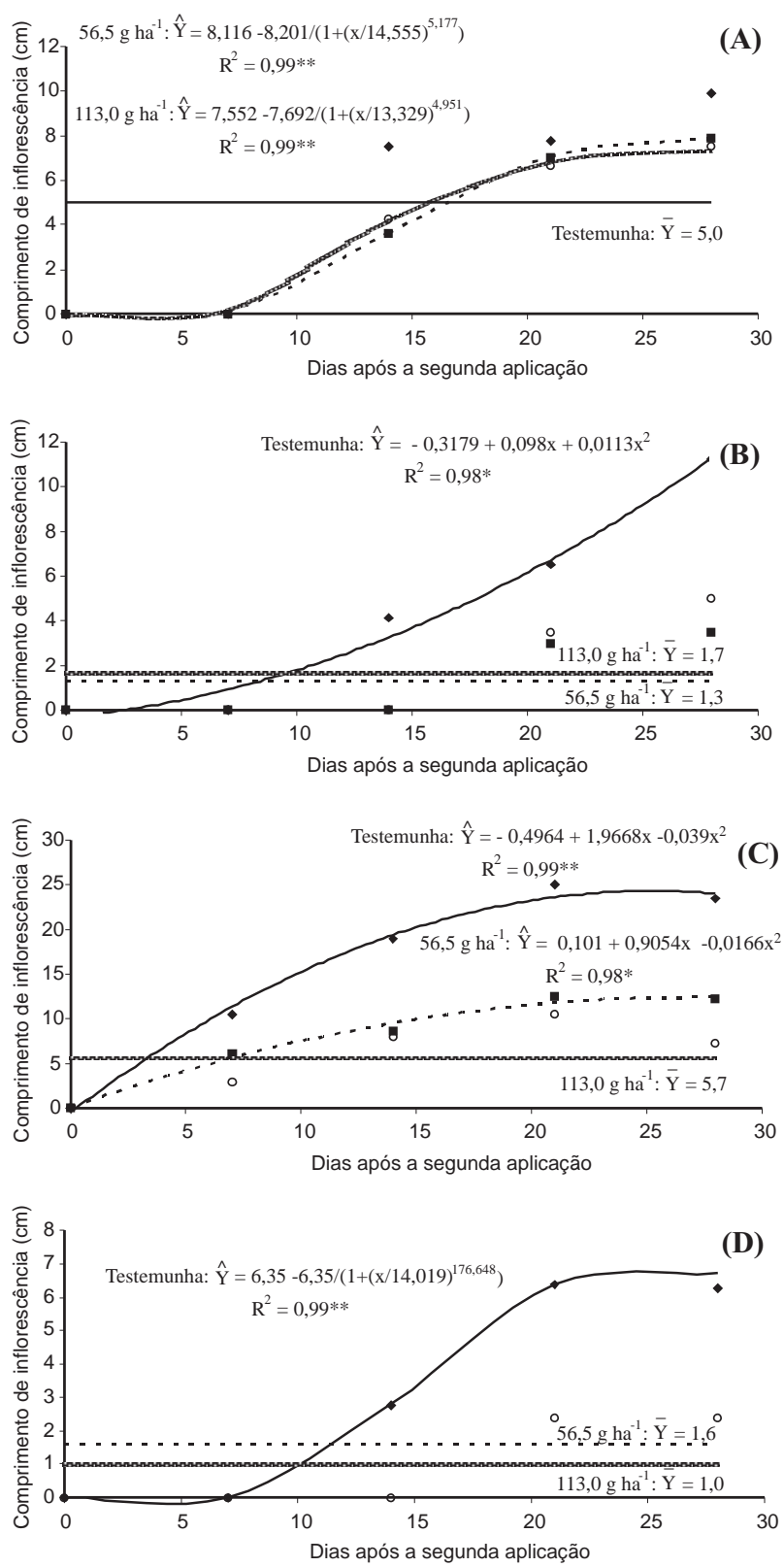

Figura 4 - Comprimento de inflorescências em diferentes épocas de avaliação, após a segunda aplicação de trinexapac-ethyl, para as espécies Axonopus compressus (A), Stenotaphrum secundatum (B), Paspalum notatum (C) e Zoysia japonica (D). Botucatu-SP - 2006. gramas, evidenciando-se plantas 38,5-40,3\%, $28,6-38,1 \%, 28,3-60,8 \%$ e $49,2-55,6 \%$ mais baixas em relação à testemunha, para as doses de 56,5 e $113,0 \mathrm{~g} \mathrm{ha}^{-1}$ de trinexapacethyl aplicadas nas espécies $A$. compressus, $S$. secundatum, $P$. notatum e $Z$. japonica, respectivamente (Tabela 1 ). A redução na estatura das plantas observada em $Z$. japonica corrobora os dados obtidos por Ervin et al. (2002), que relataram a aplicação de trinexapac-ethyl em $Z$. japonica como uma efetiva prática de manejo em áreas de baixa condição de luminosidade.

Os dados obtidos da massa seca evidenciaram menores acúmulos para $P$. notatum e $Z$. japonica, com valores de 48,5-68,7 e $65,8-66,4 \%$ para as doses de 56,5 e 113,0 $\mathrm{g} \mathrm{ha}^{-1}$ de trinexapac-ethyl, respectivamente, em relação à testemunha. Da mesma forma, A. compressus mostrou redução no acúmulo de massa seca de 36,8 e $61,8 \%$ para a menor e a maior dose utilizada do regulador vegetal, respectivamente. Somente a espécie $S$. secundatum apresentou tendência em promover diminuição acima de $40 \%$ no acúmulo de massa seca em relação à testemunha sem aplicação (Tabela 1).

Na Figura 5 estão apresentadas as equações de regressão ajustadas para os dados de altura de plantas em diferentes épocas de avaliação, após a segunda aplicação de trinexapac-ethyl nas espécies de grama avaliadas. Verificou-se que a partir dos 55 dias após a segunda aplicação do regulador vegetal, meados do mês de abril, época em que as condições de temperatura e de luminosidade não eram favoráveis ao desenvolvimento das gramas, houve tendência de paralisação do crescimento das plantas das quatro espécies avaliadas.

Pode-se notar em $A$. compressus que, para a testemunha e a menor dose de trinexapacethyl, foi constatado crescimento vegetativo na avaliação realizada aos sete dias após a segunda aplicação do regulador vegetal, ao passo que, na maior dose, observou-se incremento no crescimento vegetativo na avaliação realizada aos 21 dias após a segunda aplicação. Entretanto, o crescimento foi inferior a $1 \mathrm{~cm}$ durante todo o período de avaliação do experimento. 

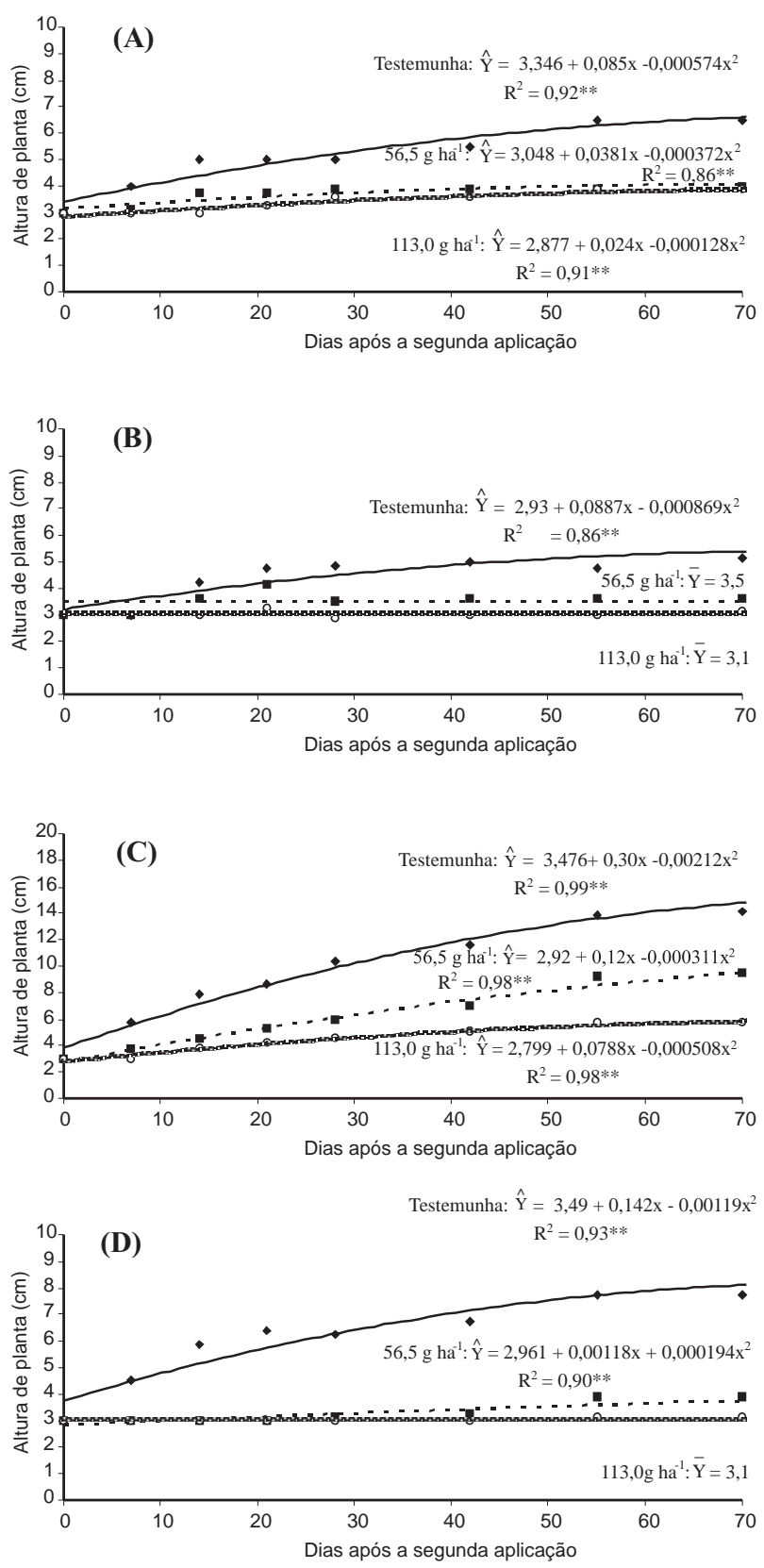

Figura 5 - Altura de plantas em diferentes épocas de avaliação, para as espécies Axonopus compressus (A), Stenotaphrum secundatum (B), Paspalum notatum (C) e Zoysia japonica (D). Botucatu-SP - 2006.

Da mesma maneira, para $S$. secundatum, a testemunha, na menor dose de trinexapacethyl, iniciou o crescimento vegetativo aos 14 dias após a segunda aplicação do regulador vegetal; contudo, a altura na menor dose do regulador vegetal foi inferior a $1 \mathrm{~cm}$ durante todo o período de avaliação, enquanto na maior dose não houve crescimento do gramado, permanecendo na altura do corte até o fim do experimento.

Já para $Z$. japonica, a testemunha iniciou o crescimento vegetativo aos sete dias após a segunda aplicação de trinexapac-ethyl. Entretanto, as doses de 56,5 e 113,0 $\mathrm{g} \mathrm{ha}^{-1}$ do regulador vegetal retardaram o crescimento do gramado em aproximadamente 55 dias após a segunda aplicação. O trinexapac-ethyl foi menos eficiente no retardamento do crescimento vegetativo da espécie $P$. notatum. No entanto, a maior dose do regulador vegetal proporcionou a maior redução da altura da grama em relação à menor dose utilizada.

Freitas et al. (2002) destacam a falta de informações detalhadas para a determinação dos niveis ideais de controle referentes às características morfológicas em gramados. Em razão disso, o autor estabeleceu para a grama Batatais ( $P$. notatum), como satisfatória, redução igual ou superior a $30 \%$ para número de inflorescência e massa seca total e de $60 \%$ para o comprimento da inflorescência, em relação à testemunha.

Com base nos niveis de controle adotados por Freitas et al. (2002), pode-se verificar no presente trabalho que a aplicação de trinexapac-ethyl foi eficiente na redução do comprimento da inflorescência e da massa seca de $P$. notatum, com valores acima de $40 \%$ para a menor dose, sendo superior a $60 \%$ para a maior dose utilizada do regulador vegetal. Entretanto, o número de inflorescência ficou acima dos niveis ideais em ambas as doses avaliadas (Tabela 1).

Do mesmo modo, Johnson (1994) determinou, em gramados de $C$. dactylon, que haveria necessidade da realização de um corte quando esta atingisse $33,3 \%$ acima da altura de corte $(3 \mathrm{~cm})$, bem como menos de $70 \%$ de redução de emissão de inflorescência.

Assim, de acordo com a recomendação de corte estipulada por Johnson (1994), que considerou a necessidade da realização de uma roçada à medida que o gramado cresce a cada $1 \mathrm{~cm}$ após o início do manejo, foi possivel determinar a necessidade de corte para as quatro espécies de gramas avaliadas no presente trabalho com base no crescimento vegetativo das testemunhas (Figura 5). Dessa maneira, 
verificou-se que para a grama $A$. compressus o trinexapac-ethyl reduziu cerca de três e cinco operações de cortes no gramado nas doses de 56,5 e $113,0 \mathrm{~g} \mathrm{ha}^{-1}$, respectivamente, uma vez que a testemunha atingiria a altura de corte a cada sete dias após cada roçada. Podese observar, ainda, que ambas as doses do regulador vegetal paralisaram o crescimento do gramado em menos de $1 \mathrm{~cm}$ de altura por todo o período de avaliação.

Para Axonopus affins, Bush et al. (1998) relatam que a dose de $0,48 \mathrm{~kg} \mathrm{ha}^{-1}$ de trinexapacethyl promoveu redução do crescimento vegetativo em $45 \%$, pelo período de seis semanas. Esses resultados diminuíram em 58\% o número de cortes requeridos pelo gramado para manter a qualidade estética.

Em $S$. secundatum, o trinexapac-ethyl reduziu em torno de quatro operações de corte no gramado durante o período da avaliação do experimento, uma vez que a testemunha atingiria a altura de corte $(4 \mathrm{~cm})$ a cada 14 dias após cada roçada, além do fato de que as doses de trinexapac-ethyl mantiveram o incremento na altura da grama em menos de $1 \mathrm{~cm}$ de altura, abaixo da necessidade de corte.

Para o gramado formado com $P$. notatum, a necessidade de realização de corte da grama pode chegar a duas operações por semana. Considerando todo o período do experimento, esse número poderia chegar a 16 operações de corte. Entretanto, a menor e a maior dose de trinexapac-ethyl reduziram por até 14 dias após a segunda aplicação do regulador vegetal a necessidade de roçada do gramado até a altura de corte de $4 \mathrm{~cm}$, totalizando economia de aproximadamente quatro operações de corte nesse período. A testemunha de $Z$. japonica apresentou altura de corte já na primeira semana após a segunda aplicação do regulador vegetal; dessa maneira, haveria a necessidade de uma operação de corte do gramado ao término desse periodo, ou seja, um corte por semana. As doses de 56,5 e 113,0 $\mathrm{g} \mathrm{ha}^{-1}$ de trinexapac-ethyl reduziram em cerca de cinco e oito operações de corte do gramado, respectivamente, entretanto, ambas as doses paralisaram o crescimento da grama em menos de $1 \mathrm{~cm}$ de altura por todo o período de avaliação.

Em termos percentuais, as doses de 56,5 e $113,0 \mathrm{~g} \mathrm{ha}^{-1}$ de trinexapac-ethyl reduziram o número de cortes nas gramas $A$. compressus, $S$. secundatum, $P$. notatum e $Z$. japonica na média de $37,5-62,5 ; 100-100 ; 12,5-12,5$ e 62,5-100\%, respectivamente, para cada espécie.

Os resultados obtidos no presente trabalho corroboram os verificados por Freitas et al. (2002) em grama $P$. notatum, que evidenciaram relação direta entre o aumento das doses de trinexapac-ethyl e a redução dos parâmetros de crescimento vegetativo e reprodutivo, sem ocorrer descoloração das folhas do gramado. É possivel evitar cortes em $P$. notatum por período de até 12 semanas com a aplicação de $0,75 \mathrm{~kg} \mathrm{ha}^{-1}$, além de promover reduções no comprimento e no número de inflorescências na ordem de 51,9 e $70,4 \%$, respectivamente, no mesmo período de avaliação.

Resultados semelhantes foram obtidos por Ferrell et al. (2003), os quais avaliaram a aplicação sequencial de trinexapac-ethyl em Paspalum vaginatum e verificaram que as doses de 0,09 e $0,38 \mathrm{~kg} \mathrm{ha}^{-1}$ proporcionaram reduções no crescimento vegetativo da grama de 59 e $96 \%$, respectivamente, por um período de 10 semanas após a aplicação. Contudo, a dose maior utilizada causou $28 \%$ de injúria na grama, enquanto a dose menor ocasionou apenas $6 \%$ de injúria. Apesar dos danos causados à qualidade visual do gramado, esses autores ressaltam a importância do uso dos reguladores vegetais no manejo de gramados, reduzindo a frequência de cortes.

Pode-se concluir que a aplicação do trinexapac-ethyl sobre as quatro espécies de gramas avaliadas demonstrou ser promissora em relação ao retardamento do crescimento vegetativo e da emissão das inflorescências, contribuindo dessa maneira no manejo desses gramados com a redução da necessidade de cortes por um período de até 55 dias após a aplicação sequencial do regulador vegetal, além de não causar efeito deletério no aspecto visual sobre as espécies de gramas avaliadas.

\section{LITERATURA CITADA}

ADAMS, R. et al. Studies on the action of the new growth retardant CGA 163935 (cimectacarb). In: KARSEN, C. M.; van LOON, L. C.; VREUGDENHIL, D. (Eds). Progress in plant growth regulation. Dordrecht: Kluwer Academic, 1992. p. $818-827$. 
BUSH, E. W. et al. Controlling growth of common carpetgrass using selected plant growth regulators. Hortscience, v. 33, n. 4 , p. 704-706, 1998.

ERVIN, E. H.; KOSKI, A. J. Trinexapac-ethyl increases Kentucky Bluegrass leaf cell density and chlorophyll concentration. Hortscience, v. 36, n. 4, p. 787-789, 2001.

ERVIN, E. H. et al. Trinexapac-ethyl restricts shoot growth and prolongs stand density of 'Meyer' zoysiagrass fairway under shade. Hortscience, v. 37, n. 3, p. 502-505. 2002.

FAGERNESS, M. J.; PENNER, D. Evaluation of V-10029 and trinexapac-ethyl for annual bluegrass seedhead suppression and growth regulation of five cool-season turfgrass species. Crop Sci., v. 12, n. 3, p. 436-440, 1998a.

FAGERNESS, M. J.; PENNER, D. Spray application parameters that influence the growth inhibiting effects of trinexapac-ethyl. Crop Sci., v. 38, n. 4, p. 1028-1035, 1998 b.

FERRELL, J. A. et al. Seashore paspalum response to trinexapac-ethyl and paclobutrazol. Hortsciense, v. 38, n. 4, p. 605-606, 2003.

FREITAS, F. C. L. et al. Efeitos do trinexapac-ethyl sobre o crescimento e florescimento da grama-batatais. Planta Daninha, v. 20, n. 3, p. 477-486, 2002.

GROSSMANN, K. et al. Regulation of plant morphology by growth retardants: Effects on phytohormone levels in soybean seedlings determined by immunoassay. Plant Physiol., v. 84, n. 4, p. 1018-1021, 1987.

JANG, H.; FRY, J. Drought responses of perennial ryegrass treated with plant growth regulators. Hotscience, v. 33, n. 2 , p. 270-273, 1998.

JOHNSON, B. J. Frequency of plant growth regulators and mowing treatments: Effects on injury and suppression of centipedegrass. Agron. J., v. 85, n. 2, p. 276-280, 1993.

JOHNSON, B. J. Influence of plant growth regulators and mowing two bermudagrasses. Agron. J., v. 86, n. 5, p. 805-810, 1994.
JOHNSON, B. J. Responde of "Tifway" bermudagrass to rate and frequency of flurprimidol and paclobutrazol application. Hotscience, v. 27, n. 3, p. 230-233, 1992.

LICKFELDT, D. W. et al. Implications of repeated trinexapac-ethyl applications on Kentuchy Bluegrass. Agron. J., v. 93, n. 5, p. 1164-1168, 2001.

MACIEL, C. D. G. et al. Comportamento vegetativo e reprodutivo da grama-batatais (Paspalum notatum Flügge) submetida à aplicação de subdoses dos herbicidas clethodim e sethoxydim. In: SIGRA - SIMPÓSIO SOBRE GRAMADOS - "ATUALIDADES \& PERSPECTIVAS", 3., 2006, Botucatu. Anais... Botucatu: FCA/Unesp, 2006. CD-ROM

NELSON, C. J.; DUNN, J. H.; COUTTS, J. H. Growth responses of tall fescue and bermudagrass to leaf applications of ancymidol. Agron. J., v. 69, n. 1, p. 61-64, 1977.

PIMENTA, C. H. Produção de gramas. In: I SIGRA SIMPÓSIO SOBRE GRAMADOS - "PRODUÇÃO, IMPLANTAÇÃO E MANUTENÇÃO”, 1., 2003, Botucatu. Anais... Botucatu: FCA/Unesp, 2003. CD-ROM.

RODRIGUES, J. D.; GODOY, L. J. G.; ONO, E. O Reguladores vegetais: bases e princípios para utilização em gramados. In: SIGRA - SIMPÓSIO SOBRE GRAMADOS - "MANEJO DE GRAMAS NA PRODUÇÃO E EM GRAMADOS FORMADOS", 2., 2004, Botucatu. Anais... Botucatu: FCA/Unesp, 2004. 30 p. CD-ROM

VELINI, E. D. Utilização de fitorreguladores em gramados. In: SIGRA - SIMPÓSIO SOBRE GRAMADOS "PRODUÇÃO, IMPLANTAÇÃO E MANUTENÇÃO", 1., 2003, Botucatu. Anais... Botucatu: FCA/Unesp, 2003. CD-ROM

WATSON, L.; DALLWITZ, M. F. The grass genera of the world. United Kingdon: CAB international: Wallingford, U. K., 1992. 1038 p. 